University of Nebraska - Lincoln

DigitalCommons@University of Nebraska - Lincoln

2019

Diisopropylammonium Bromide Based Two-Dimensional Ferroelectric Monolayer Molecular Crystal with Large In-Plane Spontaneous Polarization

Liang Ma

Yinglu Jia

Stephen Ducharme

Jinlan Wang

Xiao Cheng Zeng

Follow this and additional works at: https://digitalcommons.unl.edu/chemzeng

Part of the Analytical Chemistry Commons, Materials Chemistry Commons, and the Physical Chemistry Commons

This Article is brought to you for free and open access by the Published Research - Department of Chemistry at DigitalCommons@University of Nebraska - Lincoln. It has been accepted for inclusion in Xiao Cheng Zeng Publications by an authorized administrator of DigitalCommons@University of Nebraska - Lincoln. 


\title{
Diisopropylammonium Bromide Based Two-Dimensional Ferroelectric Monolayer Molecular Crystal with Large In-Plane Spontaneous Polarization
}

\author{
Liang Ma, ${ }^{1,2}$ Yinglu Jia, ${ }^{1}$ Stephen Ducharme, ${ }^{3}$ \\ Jinlan Wang, ${ }^{2}$ and Xiao Cheng Zeng ${ }^{1,3,4}$
}

\begin{abstract}
1 Department of Chemistry, Nebraska Center for Materials and Nanoscience, University of Nebraska-Lincoln, Lincoln, Nebraska 68588, United States 2 School of Physics, Southeast University, Nanjing 211189, China

3 Department of Physics and Astronomy, Nebraska Center for Materials and Nanoscience, University of Nebraska-Lincoln, Lincoln, Nebraska 68588, United States

4 Department of Mechanical \& Materials Engineering, University of NebraskaLincoln, Lincoln, Nebraska 68588, United States
\end{abstract}

\author{
Corresponding author - X.C. Zeng, xzeng1@unl.edu \\ ORCID \\ Liang Ma 0000-0003-4747-613X \\ Jinlan Wang 0000-0002-4529-874X \\ Xiao Cheng Zeng 0000-0003-4672-8585
}

\begin{abstract}
In light of their easy processing, light weight and mechanical flexibility, ferroelectric molecular crystal with large spontaneous polarization $\left(P_{s}\right)$ is highly desired for many advanced applications. Herein, we report the first theoretical study of two-dimensional (2D) ferroelectric molecular crystals via ab initio calculations. Specifically, we show that diisopropylammonium bromide (DIPAB) based 2D ferroelectric monolayer molecular
\end{abstract}

Published in Journal of the American Chemical Society 141 (2019), pp 1452-1456.

DOI: $10.1021 /$ jacs.8b12102

Copyright (C) 2019 American Chemical Society. Used by permission

Submitted November 15, 2018; published January 11, 2019.

Supporting Information follows the References. 


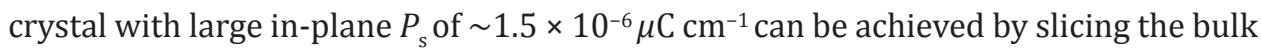
DIPAB along a specific plane while keeping the space group unchanged. The important roles of hydrogen bonds are also identified. Ab initio molecular dynamics simulations indicate that, with the support of a graphene substrate, the ferroelectric order of 2D DIPAB monolayer can be retained at room temperature. Lastly, we show that several other diisopropylammonium halide molecular crystals can also be used to achieve 2D all-organic ferroelectric monolayer singular molecular crystal with large in-plane $P_{\mathrm{s}^{*}}$.

Ferroelectricity, the spontaneous polarization $\left(\mathrm{P}_{\mathrm{s}}\right)$ that can be reversed by external field, has been widely exploited in many applications, including nonvolatile memory devices, field-effect transistors and sensors. ${ }^{1}$ Thus far, fabricated ferroelectric materials are predominately three-dimensional (3D). Recently, increasing efforts have been devoted to the development of 2D ferroelectric materials, in light of their novel properties that may not be seen in the bulk counterparts. ${ }^{2}$ Achieving ferroelectricity will increase the variety of functional 2D materials, thereby substantially broadening their application scopes. Although such an effort in developing 2D ferroelectric materials can be traced back to about two decades ago, ${ }^{3}$ major advances have been achieved only recently ${ }^{4}$ due to the suppressed $\mathrm{P}_{s}$ as well as greatly weakened domain stability by the markedly enhanced depolarization field in 2D ferroelectrics thin film. ${ }^{1 a, 3 a, 5}$

Theoretical studies have claimed that monolayers of group-V elements (e.g., P, As, Sb, and Bi) or their binary compounds (e.g., SbN and $\mathrm{BiP}$ ) or their group-IV monochalcogenide analogues can be ferroelectric. ${ }^{6}$ Robust in-plane ferroelectricity has been experimentally confirmed in the atomically thin SnTe (an example of group-IV monochalcogenides). ${ }^{7}$ Meanwhile, 2D ferroelectric $\mathrm{CuInP}_{2} \mathrm{~S}_{6}$ with a Curie temperature $\left(T_{\mathrm{c}}\right)$ of $\sim 320 \mathrm{~K}$ has also been reported. ${ }^{8}$ Intrinsic room-temperature ferroelectricity has been theoretically predicated in $2 \mathrm{D}_{2} \mathrm{Se}_{3}$ and other $\mathrm{III}_{2}-\mathrm{VI}_{3}$ van der Waals (vdW) monolayers. ${ }^{9}$ In particular, $2 \mathrm{D}$ ferroelectric $\mathrm{In}_{2} \mathrm{Se}_{3}$ was confirmed by experiment very recently. ${ }^{10}$ Direct observation of robust out-of-plane $\mathrm{P}_{\mathrm{s}}$ in films of $\mathrm{BiFeO}_{3}$ with one-unit-cell thickness has been reported. ${ }^{11}$ Several theoretical studies even suggested existence of multiferroicity, i.e., the coexistence and coupling between ferroelectricity and/or ferroelasticity/ferromagnetism, in 2D materials. . $^{6 a, f, 12}$

The organic Rochelle salt molecular crystal reported in 1921 is the first recognized ferroelectric material. ${ }^{13}$ Molecular ferroelectrics show some distinct advantages, compared to conventional inorganic ferroelectrics, including light weight, abundant chemical diversity, mechanical 
flexibility and low cost. However, the predominant development in the field of ferroelectrics for nearly one century still centers on the inorganic materials, such as $\mathrm{BaTiO}_{3}$ (BTO) and lead zirconate titanate (PZT). ${ }^{14}$ This is due in part to the fact that most molecular ferroelectrics possess much smaller $P_{\mathrm{S}}$ and lower $T_{\mathrm{c}}$ than their inorganic counterparts. Only until recently, breakthroughs in making molecular ferroelectric crystals with large $P_{\mathrm{s}}$ (up to $23 \mu \mathrm{C} \mathrm{cm}^{-2}$ ) and high $T_{\mathrm{c}}(>300 \mathrm{~K}$ ) that are comparable to those of BTO, have been made. Well-known examples include croconic acid, supramolecular complexes, diisopropylammonium bromide (DIPAB), hybrid perovskites and organic perovskites. ${ }^{15}$

One previously proposed strategy to make 2D molecular ferroelectrics is to functionalize 2D inorganic backbone with polar organic group. ${ }^{16}$ However, to our knowledge, 2D all-organic ferroelectric molecular crystal has not been reported in the literature. In this communication, we show ab initio computation evidence of a 2D DIPAB-based molecular monolayer with large in-plane $P_{s^{\prime}}$. The all-organic monolayer can be in principle achieved from slicing bulk DIPAB along a specific plane.

The ab initio computations were performed using the density functional theory (DFT) methods, implemented in the Vienna Ab initio Simulation Package (VASP 5.4). ${ }^{17}$ The general gradient approximation (GGA) type Perdew, Burke, and Ernzerhof (PBE) functional was employed. ${ }^{18}$ The electron-ion interaction was described by the projected augmented wave (PAW) method. ${ }^{19}$ The dispersion interaction was accounted for by using the Grimme's DFT-D3 method. ${ }^{20}$ The ferroelectric polarization was evaluated by using the Berry phase scheme. ${ }^{21}$ More computational details are given in the Supporting Information (SI).

The optimized structure of the ferroelectric phase of bulk DIPAB crystal is shown in Figure 1. The symmetry analysis indicates that the space group of the ferroelectric phase of bulk DIPAB is the polar $P 2_{1}$, consistent with the experimental measurement. ${ }^{15 \mathrm{~d}}$ The computed $P_{\mathrm{s}}$ of DIPAB is $22.1 \mu \mathrm{C} \mathrm{cm}^{-2}$, in good agreement with the experimental value of 23 $\mu \mathrm{C} \mathrm{cm}^{-2}$, which strongly validates our computation scheme. ${ }^{15 \mathrm{~h}}$ The ferroelectric-paraelectric transition mechanism is experimentally identified to be the order-disorder type. Upon elevating the temperature above $T_{\mathrm{c}}(\sim 426 \mathrm{~K})$, the $-\mathrm{NH}_{2}-$ groups and adjacent atoms in DIPAB molecules randomly occupy two possible mirror positions with $50 \%$ to $50 \%$ probability (Figure S1). This order-disorder transition turns the DIPAB molecular crystal into a centrosymmetric structure with the space group of $P 2_{1} / \mathrm{m}$, which cancels the $P_{\mathrm{s}}$ and yields the paraelectric phase. 

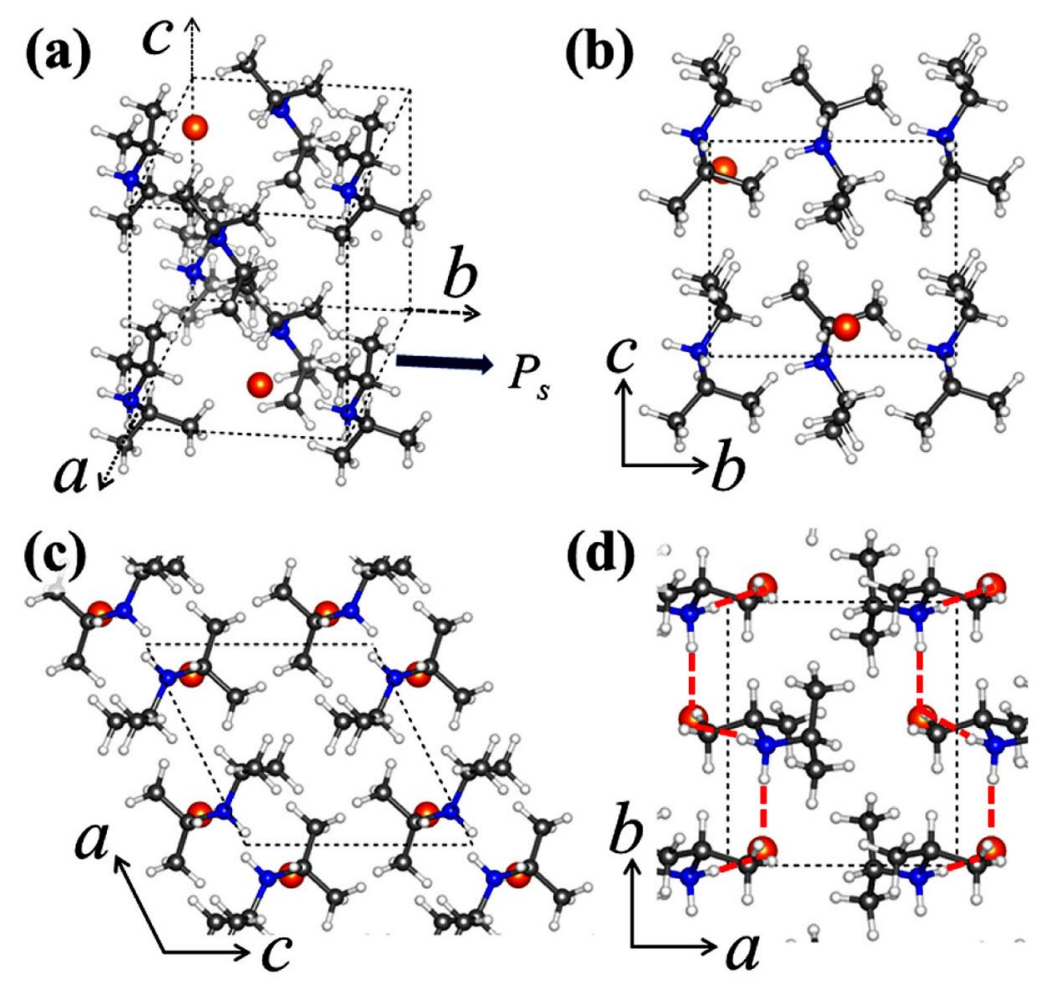

Figure 1. (a) Optimized crystal structure of ferroelectric phase of bulk DIPAB. The bold arrow denotes the Ps. Panels b, c and d show the atomic views of ferroelectric phase of DIPAB along a, b and c axis, respectively. The white, gray, blue and orange balls represent the $\mathrm{H}, \mathrm{C}, \mathrm{N}$ and $\mathrm{Br}$ atoms, respectively. The red dashed lines in panel d denote the hydrogen bonds between $\mathrm{H}$ and $\mathrm{Br}$ atoms. Figure 2. An illustration of slicing the bulk DIPAB crystal into monolayers along the (a) (001), (b) (100) and (c) (010) planes, respectively.

By closely inspecting the structure of the ferroelectric DIPAB crystal, we note that strong hydrogen-bonds are formed between $\mathrm{Br}$ and $\mathrm{H}$ atoms and are aligned in an armchair chain of $-[\mathrm{NH}]-\mathrm{H} \cdots \mathrm{Br} \cdots \mathrm{H}-[\mathrm{NH}]-$ along the $\mathrm{b}$ axis (see Figure $1 \mathrm{~d}$ ). This is due to that each $-\left[\mathrm{NH}_{2}\right]^{+}$group can provide two equivalent $\mathrm{H}$ atoms with $\mathrm{H}-\mathrm{N}-\mathrm{H}$ bond angle of $\sim 107^{\circ}$ and each $\mathrm{Br}$ atom can be shared by the $-\left[\mathrm{NH}_{2}\right]^{+}$groups of two adjacent DIPAB molecules. Such hydrogen-bonded chains can align the DIPAB molecules to pack in an ordered manner, thereby giving rise to the $P_{\mathrm{s}}$ by aligning the intrinsic dipole moment of DIPAB molecules along the $b$ axis. The polarization can be reversed/switched by transforming the armchair chain of hydrogen bonds into a mirror shape. 

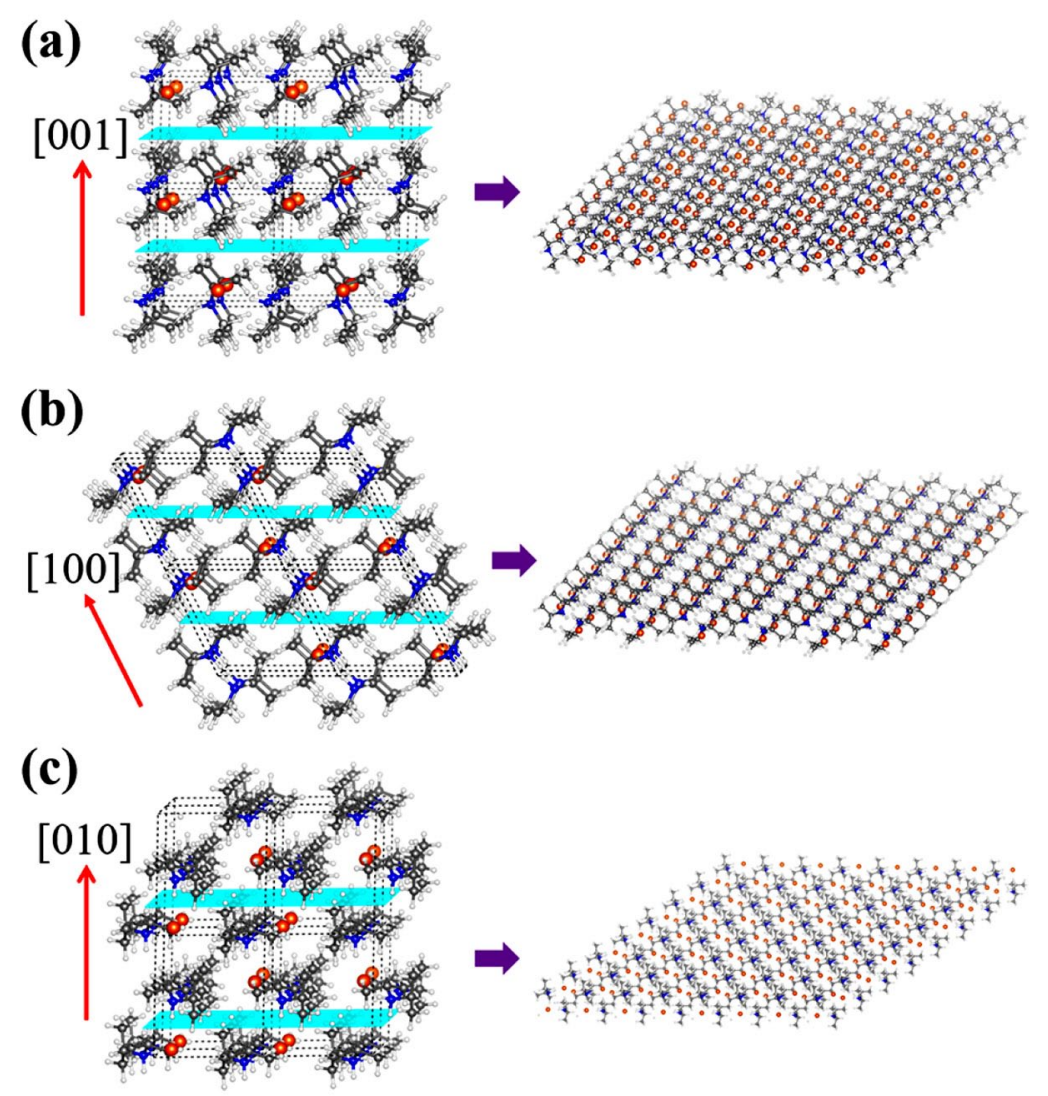

Figure 2. An illustration of slicing the bulk DIPAB crystal into monolayers along the (a) (001), (b) (100) and (c) (010) planes, respectively.

One possible way to achieve 2D DIPAB ferroelectric monolayer is to slice the bulk DIPAB molecular crystal along specific plane while keep the hydrogen-bonded chains intact. As seen from Figure 2, slicing the bulk DIPAB molecular crystal into 2D monolayers along the (001) and (100) planes can well retain the hydrogen-bonded chains. While, slicing the bulk DIPAB along the (010) plane will break the hydrogen-bonded chains in the vertical direction, which is energetically more challenging. Moreover, symmetry analysis indicates that the 2D (001) and (100) DIPAB monolayers belong to the same polar $P 2_{1}$ space group as their 3D ferroelectric matrix. Thus, large switchable in-plane $P_{\mathrm{s}}$ is also expected in both slicing cases. On the other hand, the symmetry of the 2D (010) monolayer turns into a trivial $P 1$ space group. Although the polarization along the out-of-plane direction is still expected due to the intrinsic 
Table 1. Calculated Lattice Parameters and $P_{s}$ of 2D DIPAB (001), (100) and (010) Monolayers, Respectively

\begin{tabular}{llll} 
Monolayer & $(001)$ & $(100)$ & $(010)$ \\
\hline$a(\AA)$ & 7.45 & 7.54 & 7.51 \\
$b(\AA)$ & 7.70 & .37 & 7.34 \\
$\gamma(\mathrm{deg})$ & 90.00 & 90.00 & 115.97 \\
$P_{\mathrm{s}}\left(10^{-6} \mu \mathrm{C} \mathrm{cm}^{-1}\right)$ & 1.53 & 1.54 & 0.20 \\
\hline
\end{tabular}

dipole moment of DIPAB molecules, such a polarization can be greatly lowered because of the vertical depolarization field caused by the anion-rich side and the opposite cation-rich side.

It is shown that both 2D DIPAB (001) and (100) monolayers exhibit large $\mathrm{P}_{s}$ around $\sim 1.5 \times 10^{-6} \mu \mathrm{C} \mathrm{cm}^{-1}$, respectively (Table 1), which are comparable to that of a previously predicated 2D inorganic ferroelectric monolayer of $\mathrm{SnSe}^{6 a, e}$ or $-\mathrm{CH}_{2} \mathrm{~F}$ polar group functionalized germanene. ${ }^{16 \mathrm{~d}}$ In contrast, the $\mathrm{P}_{\mathrm{s}}$ of 2D DIPAB (010) monolayer is much less than those of 2D (001) and (100) monolayers, consistent with our discussion above.

Because the 2D DIPAB monolayer may be potentially obtained by slicing the bulk DIPAB along specific plane, we estimate the cleavage energy of 2D DIPAB monolayer to confirm feasibility of getting a monolayer DIPAB via either exfoliation or mechanical peeling. The cleavage energy $(\triangle E)$ of a 2D DIPAB monolayer along a specific plane can be calculated based on the equation of $\Delta E=\left(E_{\text {layer-d }}-E_{\text {bulk }}\right) / 2 \mathrm{~S}$, where the $E_{\text {layer-d }}$ is the energy of DIPAB monolayer separated with a distance $d$ from neighboring monolayers, and $E_{\text {bulk }}$ is the energy of bulk DIPAB $(d=0 \AA)$, the $2 \mathrm{~S}$ is the surface area of the two fractured sections from both sides of the DIPAB monolayer.

As shown in Figure 3, the cleavage energy required to slice the DIPAB along (010) plane is the largest one $\left(0.17 \mathrm{~J} \mathrm{~m}^{-2}\right)$ due to the break of hydrogen-bonded chains. The cleavage energy is $\sim 0.08$ and $\sim 0.10 \mathrm{~J} \mathrm{~m}^{-2}$ for slicing DIPAB along (001) and (100) plane, respectively. The (001) case is slightly advantage compared to the (100) case. This can be understood that bulk DIPAB along (001) plane has a smaller gap between adjacent layers, giving rise to a clear section (Figure 1a). While a small part of molecules across the (100) section in a staggered way (Figure 1d), which slightly enhances the vdW interaction between molecules. Note that cleavage energy of slicing bulk DIPAB into $2 \mathrm{D}$ monolayers is only about $1 / 4$ to $1 / 5$ of that of slicing graphite into graphene $\left(\sim 0.36 \mathrm{~J} \mathrm{~m}^{-2}\right)^{22}$ 


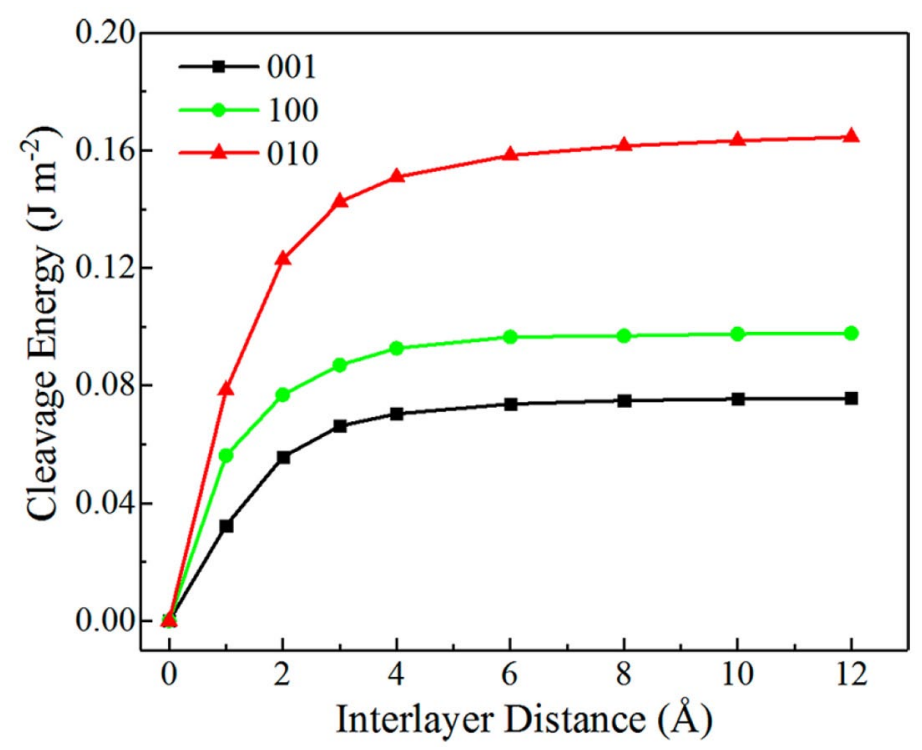

Figure 3. Cleavage energy estimation regarding the slicing of DIPAB along (001), (100) and (010) plane, respectively, by increasing the interlayer distances refer to the bulk DIPAB (0 Å). Figure 4. (a) Top and (b) side views of snapshot of the 2D DIPAB (001) monolayer supported on a graphene sheet, after 5 ps of AIMD simulation in the NPT ensemble. Note that the substrate in panel a is removed for clarity.

This indicates that slicing the bulk DIPAB into 2D monolayers along specific plane is highly feasible. Because of the lowest cleavage energy required, the 2D DIPAB (001) monolayer is expected to be the most easily cleaved, under the well-controlled experimental conditions.

Note that a chemically inert substrate or an interface, such as graphene or hexagonal boron nitride, is often used to support or stabilize monolayer molecular crystals, ${ }^{23}$ as well as $2 \mathrm{D}$ inorganic ferroelectric materials. ${ }^{7}$ Here, we also consider this possibility. To this end, we performed an ab initio molecular dynamics (AIMD) simulation in the constant temperature $(300 \mathrm{~K})$ and pressure $(0 \mathrm{GPa})(\mathrm{NPT})$ ensemble to examine thermal stability of 2D DIPAB (001) and (100) monolayer molecular crystals supported on a graphene substrate (see part II of SI for details). As shown in Figure 4, the orderly packed molecular crystal structure of 2D DIPAB (001) monolayer is maintained after 5 ps AIMD simulation (time step: $1 \mathrm{fs}$ ), suggesting thermal stability of graphene-supported a 2D DIPAB (001) monolayer at room temperature. Similar stability behavior is seen for the 2D DIPAB (100) monolayer (Figure S2). 
(a)

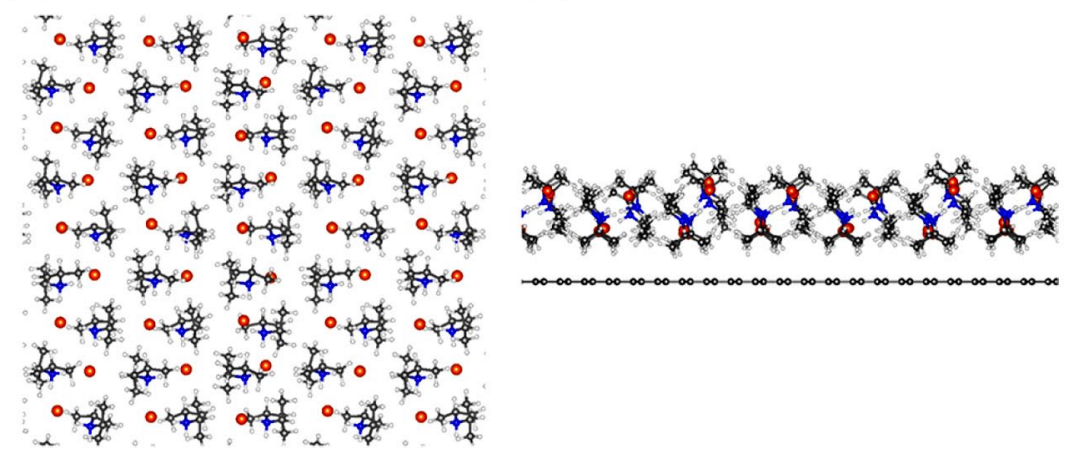

Figure 4. (a) Top and (b) side views of snapshot of the 2D DIPAB (001) monolayer supported on a graphene sheet, after 5 ps of AIMD simulation in the NPT ensemble. Note that the substrate in panel a is removed for clarity.

Lastly, we also consider two other crystalline systems of DIPAC and DIPAI, by replacing the $\mathrm{Br}^{-}$ion with either $\mathrm{Cl}^{-}$or $\mathrm{I}^{-}$ion. As the ion radius follows the order of $\mathrm{Cl}^{-}<\mathrm{Br}^{-}<\mathrm{I}^{-}$, the DIPAC exhibits a smaller lattice constant while DIPAI exhibits a larger lattice constant than that of DIPAB (see Table S1, Figures S3 and S4). The calculated $\mathrm{P}_{\mathrm{s}}$ values are 23.3 and $20.9 \mu \mathrm{C} \mathrm{cm}^{-2}$, respectively, for the bulk DIPAC and DIPAI. ${ }^{15 \mathrm{~h}}$ The cleavage energies for DIPAC or DIPAI along specific planes show the same order as DIPAB, i.e., $(001)<(100)<(010)$ (Figures S5 and S6). The cleavage energies required to break the (010) hydrogen-bonded chains are 0.18 and $0.14 \mathrm{~J} \mathrm{~m}^{-2}$ for the DIPAC and DIPAI, respectively. Notably, the (010) cleavage energy follows the order of DIPAC > DIPAB > DIPAI. This trend seems correlated with the strength order of hydrogen bond between $\mathrm{H}$ atom and halogen atom, or the electronegativity order of $\mathrm{Cl}>\mathrm{Br}>\mathrm{I}$. The calculated $\mathrm{P}_{\mathrm{s}}$ of 2D (001) and (100) monolayers of DIPAC and DIPAI are $1.61,1.54,1.47$ and $1.61 \times 10^{-6} \mu \mathrm{C} \mathrm{cm}^{-1}$ (Table S2), respectively, very close to those of the 2D DIPAB (001) and (100) monolayer.

In conclusion, we show ab initio computation evidence of DIPAB based 2D ferroelectric molecular crystal that can be achieved by slicing the bulk DIPAB along specific plane. The 2D DIPAB ferroelectric monolayer can entail high in-plane $\mathrm{P}_{\mathrm{s}}$ of $\sim 1.5 \times 10^{-6} \mu \mathrm{C} \mathrm{cm}^{-1}$, comparable to that of $2 \mathrm{D}$ inorganic ferroelectrics materials previously reported. The estimated low cleave energy of 2D DIPAB monolayers along specific plane suggest the feasibility of slicing bulk DIPAB into 2D monolayer. $A b$ initio 
molecular dynamics simulations suggest the room-temperature thermal stability of ferroelectric order of graphene-supported 2D DIPAB monolayer. Other diisopropylammonium halide molecular crystals also show similar physical features. We hope the predicted novel properties of monolayer DIPAB can motivate future experimental efforts in the synthesis of 2D all-organic ferroelectric molecular crystals.

Supporting Information - Computational details (PDF) — follows the References.

Acknowledgments This work is supported by the National Science Foundation (NSF) through the Nebraska Materials Research Science and Engineering Center (MRSEC) (grant no. DMR-1420645), the National Key Research and Development Program of China (2017YFA0204800), an NSF EPSCoR Track 2 grant (OIA- 1538893), and by the University of Nebraska Holland Computing Center.

The authors have declared there are no competing financial interests.

\section{References}

(1) (a) Dawber, M.; Rabe, K. M.; Scott, J. F. Physics of Thin-Film Ferroelectric Oxides. Rev. Mod. Phys. 2005, 77, 1083-1130. (b) Scott, J. F. Applications of Modern Ferroelectrics. Science 2007, 315, 954- 959. (c) Martin, L. W.; Rappe, A. M. Thin-Film Ferroelectric Materials and Their Applications. Nat. Rev. Mater. 2017, 2, 16087 .

(2) Novoselov, K. S.; Mishchenko, A.; Carvalho, A.; Castro Neto, A. H. 2D Materials and van der Waals Heterostructures. Science 2016, 353, aac9439.

(3) (a) Bune, A. V.; Fridkin, V. M.; Ducharme, S.; Blinov, L. M.; Palto, S. P.; Sorokin, A. V.; Yudin, S. G.; Zlatkin, A. Two-Dimensional Ferroelectric Films. Nature 1998, 391, 874-877. (b) Blinov, L. M.; Fridkin, V. M.; Palto, S. P.; Bune, A. V.; Dowben, P. A.; Ducharme, S. Two-Dimensional Ferroelectrics. Phys.-Usp. 2000, 43, 243-257.

(4) Cui, C.; Xue, F.; Hu, W.-J.; Li, L.-J. Two-Dimensional Materials with Piezoelectric and Ferroelectric Functionalities. npj 2D Mater. Appl. 2018, 2, 18.

(5) Kittel, C. Thickness of Domain Walls in Ferroelectric and Ferroelastic Crystals. Solid State Commun. 1972, 10, 119-121.

(6) (a) Wu, M.; Zeng, X. C. Intrinsic Ferroelasticity and/or Multiferroicity in Two-Dimensional Phosphorene and Phosphorene Analogues. Nano Lett. 2016, 16, 3236-3241. (b) Xiao, C.; Wang, F.; Yang, S. A.; Lu, Y.; Feng, Y.; Zhang, S. Elemental Ferroelectricity and Antiferroelectricity in Group-V Monolayer. Adv. Funct. 
Mater. 2018, 28, 1707383. (c) Liu, C.; Wan, W.; Ma, J.; Guo, W.; Yao, Y. Robust Ferroelectricity in Two-Dimensional SbN and BiP. Nanoscale 2018, 10, 7984-7990. (d) Hanakata, P. Z.; Carvalho, A.; Campbell, D. K.; Park, H. S. Polarization and Valley Switching in Monolayer Group-IV Monochalcogenides. Phys. Rev. B: Condens. Matter Mater. Phys. 2016, 94, 035304. (e) Fei, R.; Kang, W.; Yang, L. Ferroelectricity and Phase Transitions in Monolayer Group-IV Monochalcogenides. Phys. Rev. Lett. 2016, 117, 097601. (f) Wang, H.; Qian, X. Two-Dimensional Multiferroics in Monolayer Group IV Monochalcogenides. 2D Mater. 2017, 4, 015042.

(7) Chang, K.; Liu, J.; Lin, H.; Wang, N.; Zhao, K.; Zhang, A.; Jin, F.; Zhong, Y.; Hu, X.; Duan, W.; Zhang, Q.; Fu, L.; Xue, Q.-K.; Chen, X.; Ji, S.-H. Discovery of Robust inPlane Ferroelectricity in Atomic- Thick SnTe. Science 2016, 353, 274-278.

(8) Liu, F.; You, L.; Seyler, K. L.; Li, X.; Yu, P.; Lin, J.; Wang, X.; Zhou, J.; Wang, H.; He, H.; Pantelides, S. T.; Zhou, W.; Sharma, P.; Xu, X.; Ajayan, P. M.; Wang, J.; Liu, Z. RoomTemperature Ferroelectricity in $\mathrm{CuInP}_{2} \mathrm{~S}_{6}$ Ultrathin Flakes. Nat. Commun. 2016, 7, 12357.

(9) Ding, W.; Zhu, J.; Wang, Z.; Gao, Y.; Xiao, D.; Gu, Y.; Zhang, Z.; Zhu, W. Prediction of Intrinsic Two-Dimensional Ferroelectrics in $\mathrm{In}_{2} \mathrm{Se}_{3}$ and Other $\mathrm{III}_{2}-\mathrm{VI}_{3}$ van der Waals Materials. Nat. Commun. 2017, 8, 14956.

(10) (a) Xiao, J.; Zhu, H.; Wang, Y.; Feng, W.; Hu, Y.; Dasgupta, A.; Han, Y.; Wang, Y.; Muller, D. A.; Martin, L. W.; Hu, P.; Zhang, X. Intrinsic Two-Dimensional Ferroelectricity with Dipole Locking. Phys. Rev. Lett. 2018, 120, 227601. (b) Cui, C.; Hu, W.-J.; Yan, X.; Addiego, C.; Gao, W.; Wang, Y.; Wang, Z.; Li, L.; Cheng, Y.; Li, P.; Zhang, X.; Alshareef, H. N.; Wu, T.; Zhu, W.; Pan, X.; Li, L.-J. Intercorrelated inPlane and out-of-Plane Ferroelectricity in Ultrathin Two-Dimensional Layered Semiconductor $\operatorname{In}_{2} \mathrm{Se}_{3}$. Nano Lett. 2018, 18, 1253-1258.

(11) Wang, H.; Liu, Z. R.; Yoong, H. Y.; Paudel, T. R.; Xiao, J. X.; Guo, R.; Lin, W. N.; Yang, P.; Wang, J.; Chow, G. M.; Venkatesan, T.; Tsymbal, E. Y.; Tian, H.; Chen, J. S. Direct Observation of Room-Temperature out-of-Plane Ferroelectricity and Tunneling Electroresistance at the Two-Dimensional Limit. Nat. Commun. 2018, 9, 3319.

(12) (a) Huang, C.; Du, Y.; Wu, H.; Xiang, H.; Deng, K.; Kan, E. Prediction of Intrinsic Ferromagnetic Ferroelectricity in a Transition- Metal Halide Monolayer. Phys. Rev. Lett. 2018, 120, 147601. (b) Zhao, Y.; Lin, L.; Zhou, Q.; Li, Y.; Yuan, S.; Chen, Q.; Dong, S.; Wang, J. Surface Vacancy-Induced Switchable Electric Polarization and Enhanced Ferromagnetism in Monolayer Metal Trihalides. Nano Lett. 2018, 18, 2943-2949. (c) Chandrasekaran, A.; Mishra, A.; Singh, A. K. Ferroelectricity, Antiferroelectricity, and Ultrathin 2D Electron/Hole Gas in Multifunctional Monolayer Mxene. Nano Lett. 2017, 17, 3290-3296. (d) Zhang, J.-J.; Lin, L.; Zhang, Y.; Wu, M.; Yakobson, B. I.; Dong, S. Type-II Multiferroic $\mathrm{Hf}_{2} \mathrm{VC}_{2} \mathrm{~F}_{2} \mathrm{Mx}-$ ene Monolayer with High Transition Temperature. J. Am. Chem. Soc. 2018, 140, 9768-9773.

(13) Valasek, J. Piezo-Electric and Allied Phenomena in Rochelle Salt. Phys. Rev. 1921, 17, 475-481.

(14) (a) Tayi, A. S.; Kaeser, A.; Matsumoto, M.; Aida, T.; Stupp, S. I. Supramolecular Ferroelectrics. Nat. Chem. 2015, 7, 281-294. (b) Horiuchi, S.; Tokura, Y. Organic Ferroelectrics. Nat. Mater. 2008, 7, 357-366. 
(15) (a) Horiuchi, S.; Tokunaga, Y.; Giovannetti, G.; Picozzi, S.; Itoh, H.; Shimano, R.; Kumai, R.; Tokura, Y. Above-Room- Temperature Ferroelectricity in a SingleComponent Molecular Crystal. Nature 2010, 463, 789-792. (b) Tayi, A. S.; Shveyd, A. K.; Sue, A. C. H.; Szarko, J. M.; Rolczynski, B. S.; Cao, D.; Kennedy, T. J.; Sarjeant, A. A.; Stern, C. L.; Paxton, W. F.; Wu, W.; Dey, S. K.; Fahrenbach, A. C.; Guest, J. R.; Mohseni, H.; Chen, L. X.; Wang, K. L.; Stoddart, J. F.; Stupp, S. I. RoomTemperature Ferroelectricity in Supramolecular Networks of Charge-Transfer Complexes. Nature 2012, 488, 485-489. (c) Chen, S.; Zeng, X. C. Design of Ferroelectric Organic Molecular Crystals with Ultrahigh Polarization. J. Am. Chem. Soc. 2014, 136, 6428-6436. (d) Fu, D.-W.; Cai, H.-L.; Liu, Y.; Ye, Q.; Zhang, W.; Zhang, Y.; Chen, X.-Y.; Giovannetti, G.; Capone, M.; Li, J.; Xiong, R.-G. Diisopropylammonium Bromide is a High-Temperature Molecular Ferroelectric Crystal. Science 2013, 339, 425-428. (e) You, Y.-M.; Liao, W.-Q.; Zhao, D.; Ye, H.-Y.; Zhang, Y.; Zhou, Q.; Niu, X.; Wang, J.; Li, P.-F.; Fu, D.-W.; Wang, Z.; Gao, S.; Yang, K.; Liu, J.M.; Li, J.; Yan, Y.; Xiong, R.-G. An Organic-Inorganic Perovskite Ferroelectric with Large Piezoelectric Response. Science 2017, 357, 306-309. (f) Ye, H.-Y.; Tang, Y.Y.; Li, P.-F.; Liao, W.-Q.; Gao, J.-X.; Hua, X.-N.; Cai, H.; Shi, P.-P.; You, Y.-M.; Xiong, R.G. Metal-Free Three-Dimensional Perovskite Ferroelectrics. Science 2018, 361, 151-155. (g) Lu, H.; Li, T.; Poddar, S.; Goit, O.; Lipatov, A.; Sinitskii, A.; Ducharme, S.; Gruverman, A. Statics and Dynamics of Ferroelectric Domains in Diisopropylammonium Bromide. Adv. Mater. 2015, 27, 7832-7838. (h) Louis, L.; Pitike, K. C.; Ghosh, A.; Poddar, S.; Ducharme, S.; Nakhmanson, S. M. Polarization Canting in Ferroelectric Diisopropylammonium-Halide Molecular Crystals: A Computational First Principles Study. J. Mater. Chem. C 2018, 6, 1143-1152.

(16) (a) Nakhmanson, S. M.; Nardelli, M. B.; Bernholc, J. Ab Initio Studies of Polarization and Piezoelectricity in Vinylidene Fluoride and Bn-Based Polymers. Phys. Rev. Lett. 2004, 92, 115504. (b) Kan, E.; Wu, F.; Deng, K.; Tang, W. High-Temperature Ferro-Electricity in Two-Dimensional Atomic Crystal. Appl. Phys. Lett. 2013, 103, 193103. (c) Wu, M.; Burton, J. D.; Tsymbal, E. Y.; Zeng, X. C.; Jena, P. Hydroxyl-Decorated Graphene Systems as Candidates for Organic Metal-Free Ferroelectrics, Multiferroics, and High-Performance Proton Battery Cathode Materials. Phys. Rev. B: Condens. Matter Mater. Phys. 2013, 87, 081406. (d) Wu, M.; Dong, S.; Yao, K.; Liu, J.; Zeng, X. C. Ferroelectricity in Covalently Functionalized Two- Dimensional Materials: Integration of High-Mobility Semiconductors and Nonvolatile Memory. Nano Lett. 2016, 16, 7309-7315.

(17) (a) Kresse, G.; Furthmüller, J. Efficient Iterative Schemes for Ab Initio Total-Energy Calculations Using a Plane-Wave Basis Set. Phys. Rev. B: Condens. Matter Mater. Phys. 1996, 54, 11169-11186. (b) Kresse, G.; Furthmuller, J. Efficiency of Ab-Initio Total Energy Calculations for Metals and Semiconductors Using a Plane-Wave Basis Set. Comput. Mater. Sci. 1996, 6, 15-50.

(18) Perdew, J. P.; Burke, K.; Ernzerhof, M. Generalized Gradient Approximation Made Simple. Phys. Rev. Lett. 1996, 77, 3865-3868.

(19) (a) Blöchl, P. E. Projector Augmented-Wave Method. Phys. Rev. B: Condens. Matter Mater. Phys. 1994, 50, 17953-17979.

(b) Kresse, G.; Joubert, D. From Ultrasoft Pseudopotentials to the Projector Augmented-Wave Method. Phys. Rev. B: Condens. Matter Mater. Phys. 1999, 59, 
1758-1775.

(20) Grimme, S.; Antony, J.; Ehrlich, S.; Krieg, H. A Consistent and Accurate Ab Initio Parametrization of Density Functional Dispersion Correction (DFT-D) for the 94 Elements H-Pu. J. Chem. Phys. 2010, 132, 154104.

(21) (a) King-Smith, R. D.; Vanderbilt, D. Theory of Polarization of Crystalline Solids. Phys. Rev. B: Condens. Matter Mater. Phys. 1993, 47, 1651-1654. (b) Vanderbilt, D.; King-Smith, R. D. Electric Polarization as a Bulk Quantity and Its Relation to Surface Charge. Phys. Rev. B: Condens. Matter Mater. Phys. 1993, 48, 4442-4455.

(22) Wang, W.; Dai, S.; Li, X.; Yang, J.; Srolovitz, D. J.; Zheng, Q. Measurement of the Cleavage Energy of Graphite. Nat. Commun. 2015, 6, 7853.

(23) (a) He, D.; Zhang, Y.; Wu, Q.; Xu, R.; Nan, H.; Liu, J.; Yao, J.; Wang, Z.; Yuan, S.; Li, Y.; Shi, Y.; Wang, J.; Ni, Z.; He, L.; Miao, F.; Song, F.; Xu, H.; Watanabe, K.; Taniguchi, T.; Xu, J.-B.; Wang, X. Two-Dimensional Quasi-Freestanding Molecular Crystals for High- Performance Organic Field-Effect Transistors. Nat. Commun. 2014, 5, 5162. (b) Zhao, Y.; Wu, Q.; Chen, Q.; Wang, J. Molecular Self- Assembly on TwoDimensional Atomic Crystals: Insights from Molecular Dynamics Simulations. J. Phys. Chem. Lett. 2015, 6, 4518-4524. (c) Wang, Q.; Yang, F.; Zhang, Y.; Chen, M.; Zhang, X.; Lei, S.; Li, R.; Hu, W. Space-Confined Strategy toward Large-Area TwoDimensional Single Crystals of Molecular Materials. J. Am. Chem. Soc. 2018, $140,5339-5342$. 


\title{
Supporting Information for
}

\section{Diisopropylammonium Bromide Based Two-Dimensional Ferroelectric}

\author{
Monolayer Molecular Crystal with Large In-Plane Spontaneous \\ Polarization
}

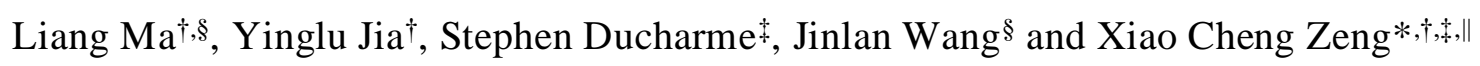

'Department of Chemistry, University of Nebraska-Lincoln, Lincoln, NE 68588, United States ${ }_{\$}$ School of Physics, Southeast University, Nanjing 211189, China

*Department of Physics and Astronomy, Nebraska Center for Materials and Nanoscience, University of Nebraska-Lincoln, Lincoln, Nebraska 68588, United States

"Department of Mechanical \& Materials Engineering, University of Nebraska-Lincoln, Lincoln, Nebraska 68588, United States

*Email: xzeng1@unl.edu 


\section{Computational Details}

Density functional theory (DFT) computations were performed by employing the projected augmented wave (PAW) potentials ${ }^{1}$ and the exchange-correlation functional based on the PerdewBurke-Ernzerhof $(\mathrm{PBE})^{2}$ functional within the generalized gradient approximation (GGA), all implemented in the Vienna ab initio simulation package (VASP 5.4). ${ }^{3}$ The periodic replica of 2D DIPAB monolayer molecular crystal along $z$ direction were separated by a vacuum space of $>15$ $\AA$ to avoid potential artificial interaction among periodic images. For the optimization of lattice and atomic position, a $6 \times 6 \times 1$ and $6 \times 6 \times 6$ Monkhorst-Pack k-point meshes were used for the Brillouin zone integration sampling of $2 \mathrm{D}$ monolayer and for the $3 \mathrm{D}$ bulk of diisopropylammonium bromide (DIPAB), diisopropylammonium chloride (DIPAC) and diisopropylammonium iodide (DIPAI) molecular crystals, respectively. A denser $9 \times 9 \times 1$ and 9 $\times 9 \times 9$ meshes were used for the calculation of electronic polarization of $2 \mathrm{D}$ and $3 \mathrm{D}$ systems, respectively. ${ }^{4}$ The initial structures of DIPAB and DIPAC were taken from the experimental reports. ${ }^{5}$ All structures were relaxed until the residual force component acting on each atom was less than $0.01 \mathrm{eV} / \mathrm{A}$. The convergence criteria for electronic relaxation was $10^{-5} \mathrm{eV}$. The energy cutoff for the plane-wave basis sets was set to be $400 \mathrm{eV}$. After careful comparison, we found that the optimized lattice parameters of bulk DIPAB crystal by using the PBE + vdW-D3 scheme ${ }^{6}$ are comparable to or even better than those by using the meta-GGA type SCAN functional ${ }^{7}+\mathrm{rVV}^{8} 0^{8}$ $\mathrm{vdW}$ density functional scheme, ${ }^{9}$ as the results are in good agreement $(\leq 3 \%)$ with the experimental results (Table S1). Due to much more computationally expensive SCAN + rVV10 method, here, only the PBE+D3 scheme was adopted for all computations.

It is known that the Berry-phase method can introduce the polarization indetermination quantum (PIQ) through multi-modulo of $n e R / V$ due to the periodic boundary condition- where $e$ is the electron charge, $R$ is a translation vector of the real-space lattice, $V$ is the unit-cell volume and $n$ is an integer. To obtain well-defined polarization, the PIQ (the $n$ ) has to be determined. In the present work, we determine the PIQ in the Berry-phase calculation of DIPAB molecular crystal by simply applying a point charge model for such an ionic system to roughly estimate the polarization. We carefully choose the unit cell of bulk DIPAB crystal so that the dipole moments will not be broken up by the cell boundaries (based on the experimental structure ${ }^{5 a}$ ). The negative charge centers are assumed at the $\mathrm{Br}^{-}$ions while the positive charge centers are assumed at the 
geometric center of two $\mathrm{H}$ atoms of the $-[\mathrm{NH} 2]^{+}$groups. We can estimate the polarization by using the equation of $P=\Sigma q^{*} r$, where $q$ is the charge (e for the $-[\mathrm{NH} 2]^{+}$group and $-\mathrm{e}$ for the $\mathrm{Br}^{-}$ion) and $r$ is the position vector of each charge center. The point charge model gives us an estimated polarization $P_{\mathrm{s}}$ of $20.77 \mu \mathrm{C} \mathrm{cm}^{-2}$ for the bulk DIPAB molecular crystal. This benchmark test value can help to determine the PIQ number $n$ in the Berry-phase calculation. The Berry-phase method finally gives a value of $P_{\mathrm{s}}$ of $22.1 \mu \mathrm{C} \mathrm{cm}{ }^{-2}$, which is in good agreement with the experimental value of $23 \mu \mathrm{C} \mathrm{cm}^{-2}$. ${ }^{5 a}$ It is worth noting that the point charge model can give a reasonable estimation of $P_{\mathrm{s}}$ for the DIPAB molecular crystal. The difference in the computed $P_{\mathrm{s}}$ between point charge model and the Berry phase method can be attributed to the over-localized point charge model and neglected electron density distribution. Such a point charge model is also used to determine the PIQ number $n$ in 2D monolayer DIPAB molecular crystal.

Table S1.Calculated lattice parameters of bulk DIPAB, DIPAC and DIPAI crystals, based on the PBE + D3 and SCAN + rVV10 schemes, and the corresponding experimental (Exp.) values.

\begin{tabular}{|c|c|c|c|c|c|c|}
\hline Lattice & $a(\AA)$ & $b(\AA)$ & $c(\AA)$ & $\alpha\left({ }^{\circ}\right)$ & $\beta\left({ }^{\circ}\right)$ & $\gamma\left({ }^{\circ}\right)$ \\
\hline \multicolumn{7}{|c|}{ DIPAB } \\
\hline $\mathrm{PBE}+\mathrm{D} 3$ & 7.55 & 7.79 & 7.62 & 90.00 & 116.54 & 90.00 \\
\hline SCAN+rVV10 & 7.49 & 7.75 & 7.56 & 90.00 & 116.65 & 90.00 \\
\hline Exp. $^{7}$ & 7.766 & 8.034 & 7.836 & 90.000 & 116.346 & 90.000 \\
\hline \multicolumn{7}{|c|}{ DIPAC } \\
\hline $\mathrm{PBE}+\mathrm{D} 3$ & 7.31 & 7.62 & 7.50 & 90.00 & 115.34 & 90.00 \\
\hline Exp. $^{8}$ & 7.674 & 7.945 & 7.766 & 90.000 & 114.950 & 90.000 \\
\hline \multicolumn{7}{|c|}{ DIPAI } \\
\hline $\mathrm{PBE}+\mathrm{D} 3$ & 7.74 & 7.93 & 7.78 & 90.000 & 117.13 & 90.00 \\
\hline
\end{tabular}




\section{Details for Ab Initio Molecular Dynamics Simulations.}

Ab initio molecular dynamics (AIMD) simulations of 2D DIPAB monolayer molecular crystal were performed in the constant temperature $(300 \mathrm{~K})$ and pressure $(0 \mathrm{GPa})(N P T)$ ensemble with the GGA-PBE functional and the D3 dispersion correction scheme, as implemented in the VASP 5.4. Both the atomic and lattice degrees of freedom were allowed to relax in the AIMD simulations, in which the Langevin thermostat ${ }^{10}$ and the Parrinello-Rahman dynamics ${ }^{11}$ were adopted. A $3 \times 3$ supercell (432 atoms) of 2D DIPAB (001) or (100) monolayer was placed on a $5 \sqrt{3} \times 9$ rectangle graphene substrate (180 atoms) to ensure the lattice mismatch less than 5\%. The graphene substrate was fixed in our AIMD simulation. To achieve the thermal equilibrium, a 5 ps AIMD simulation with time step of $1 \mathrm{fs}$ was performed for each system. 

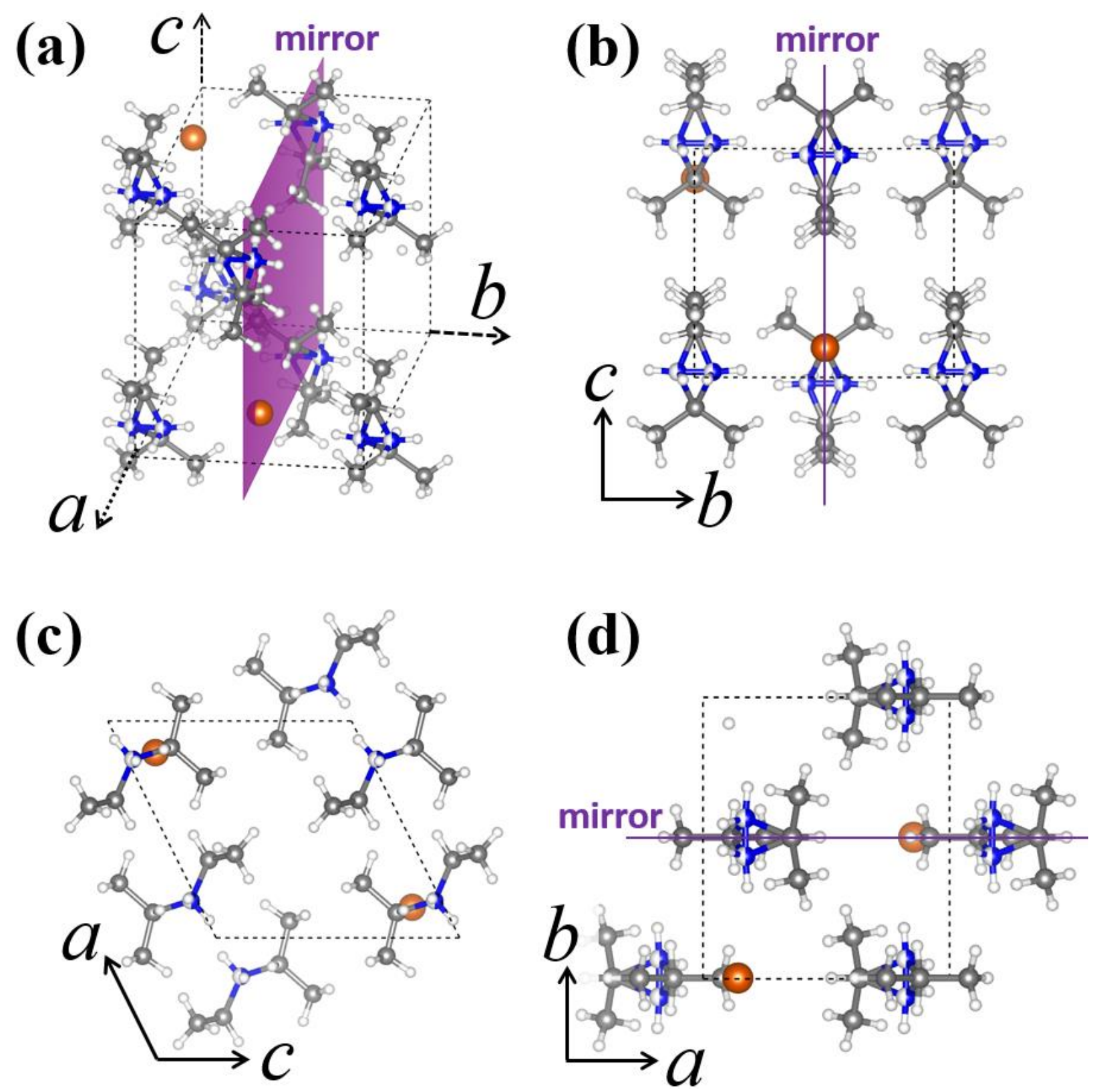

Figure S1. (a) Optimized structure of paraelectric bulk DIPAB crystal. The purple plane denotes the mirror plane of the paraelectric phase with space group $P 21 / \mathrm{m}$. (b), (c) and (d) show the atomic views of paraelectric phase of DIPAB along $a, b$ and $c$ axis, respectively. The white, grey, blue and orange balls represent the $\mathrm{H}, \mathrm{C}, \mathrm{N}$ and $\mathrm{Br}$ atoms, respectively. 
(a)

(b)
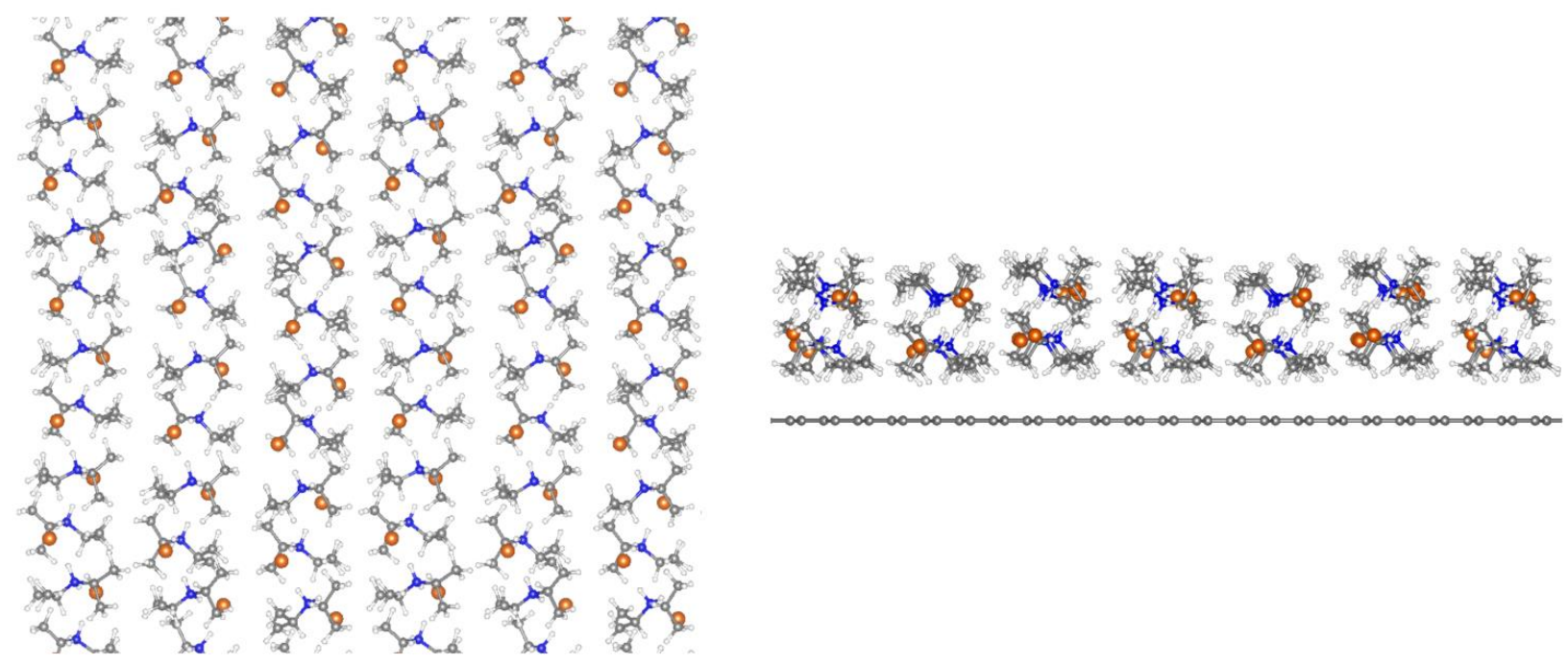

Figure S2. (a) Top and (b) side views of snapshots of the 2D DIPAB (100) monolayer after 5 ps of AIMD simulation (time step: $1 \mathrm{fs}$ ) in the $N P T$ ensemble, where the temperature is controlled at $300 \mathrm{~K}$ and pressure is controlled at $0 \mathrm{GPa}$. Note that the substrate in top view is removed for clarity. 


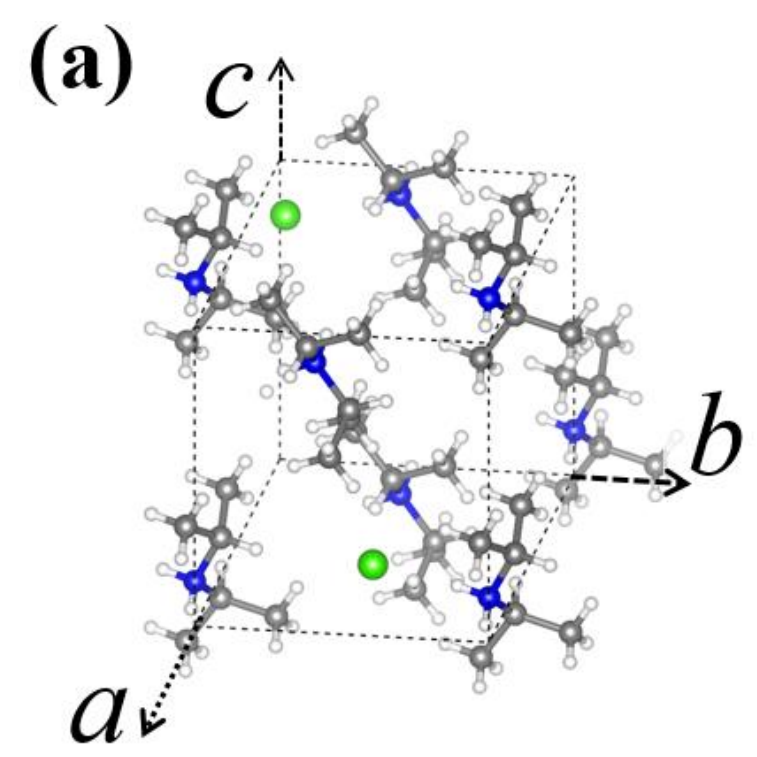

(b)
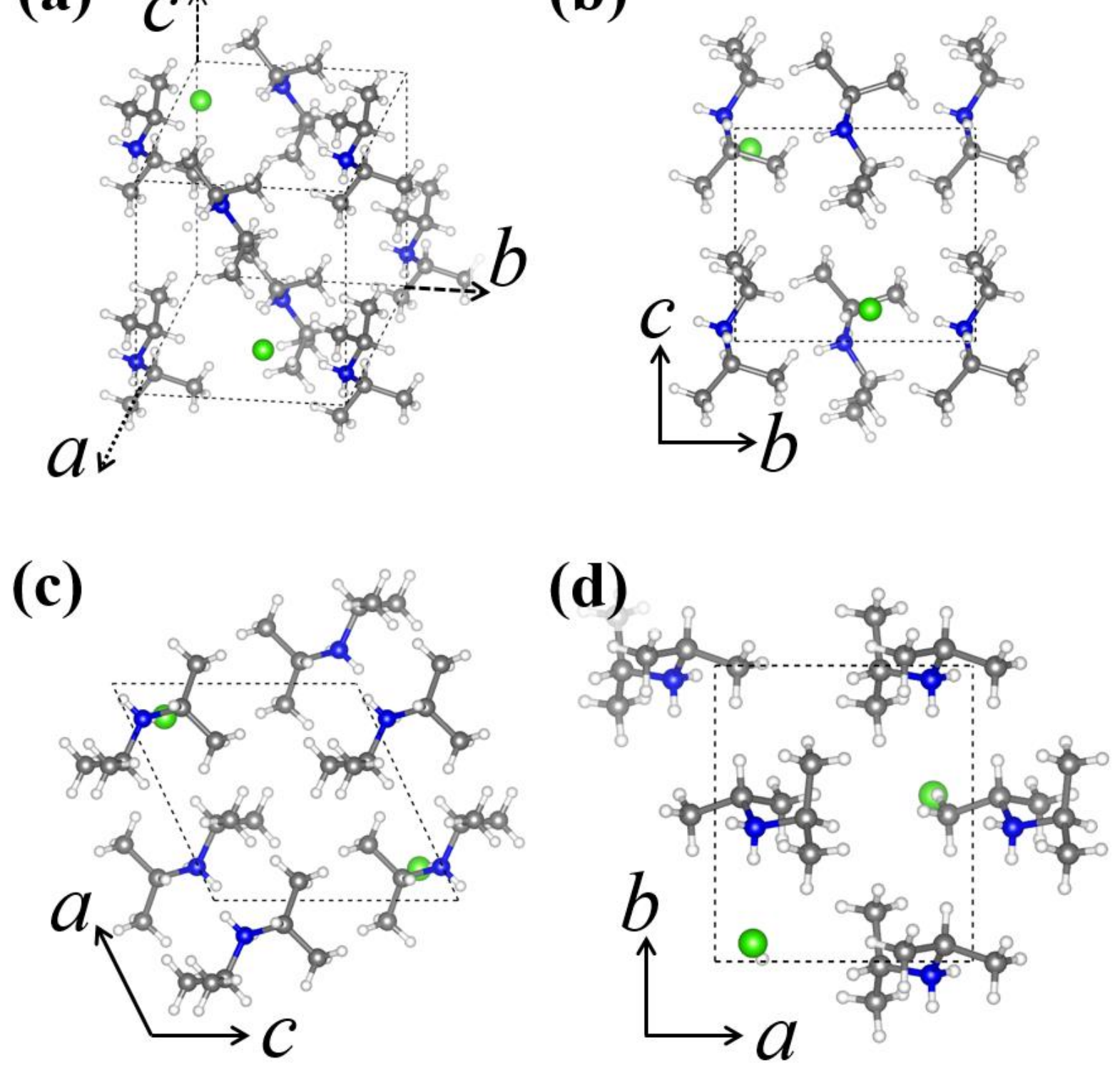

Figure S3. (a) Optimized structure of ferroelectric bulk DIPAC crystal. (b), (c) and (d) show the atomic views of ferroelectric phase of DIPAC along $a, b$ and $c$ axis, respectively. The white, grey, blue and green balls represent the $\mathrm{H}, \mathrm{C}, \mathrm{N}$ and $\mathrm{Cl}$ atoms, respectively. 


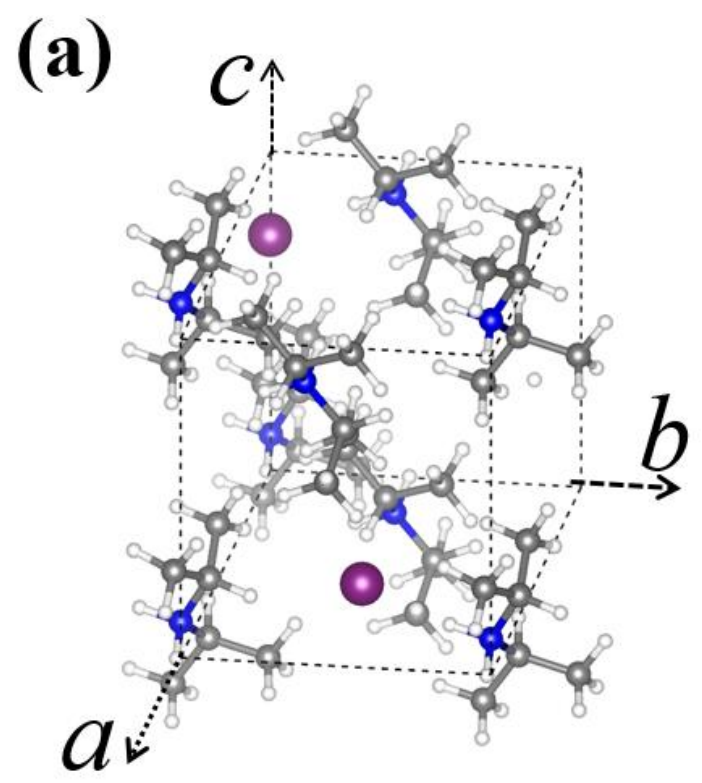

(b)
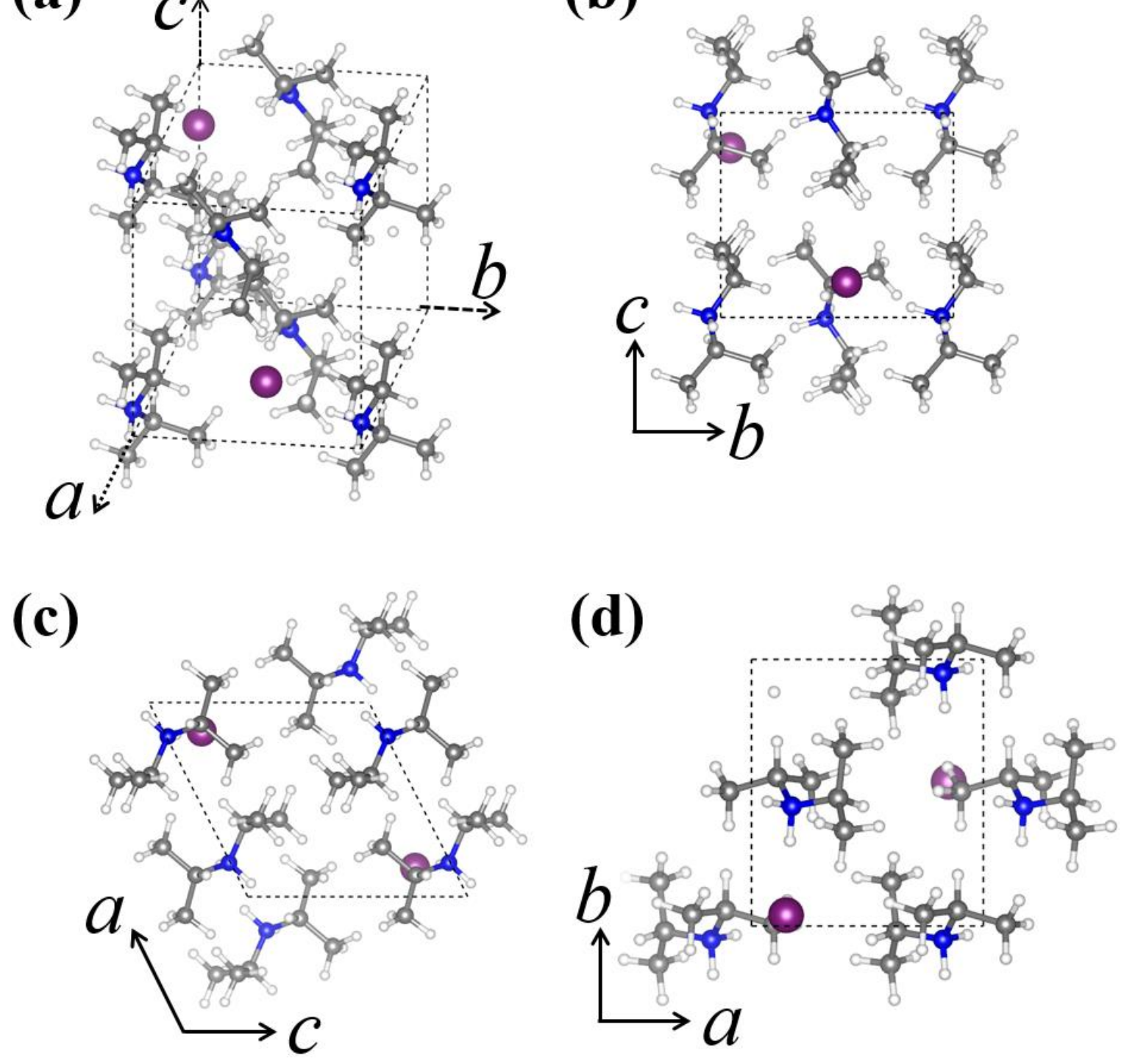

Figure S4. (a) Optimized structure of ferroelectric bulk DIPAI crystal. (b), (c) and (d) show the atomic views of the ferroelectric phase of DIPAI along $a, b$ and $c$ axis, respectively. The white, grey, blue and purple balls represent the H, C, N and I atoms, respectively. 


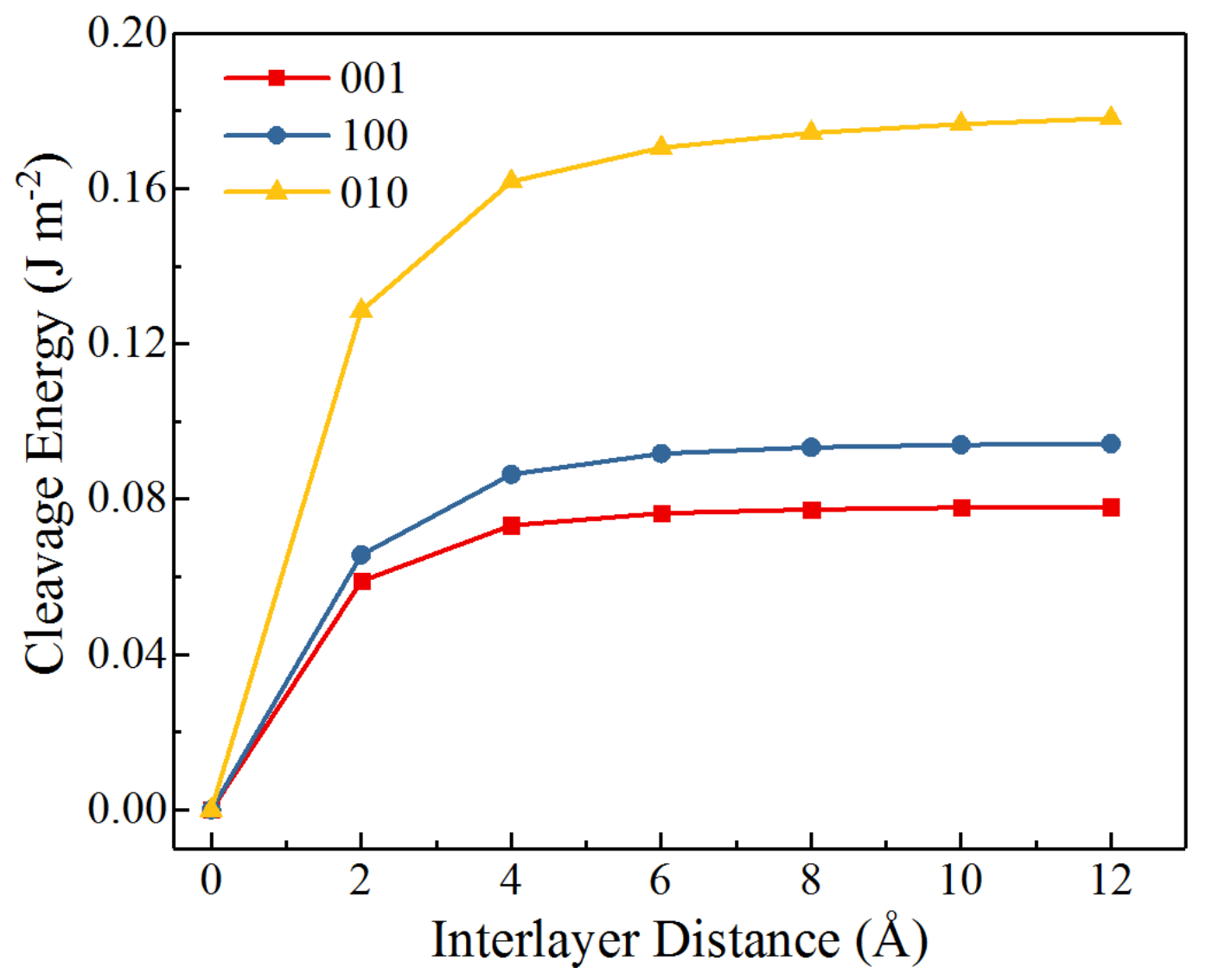

Figure S5. Cleavage energy estimation on the mechanical peeling of DIPAC along the (001), (100) and (010) planes, respectively, versus the interlayer distance. The reference starting point ( $0 \AA$ ) corresponds to the perfect ferroelectric bulk DIPAC crystal. 


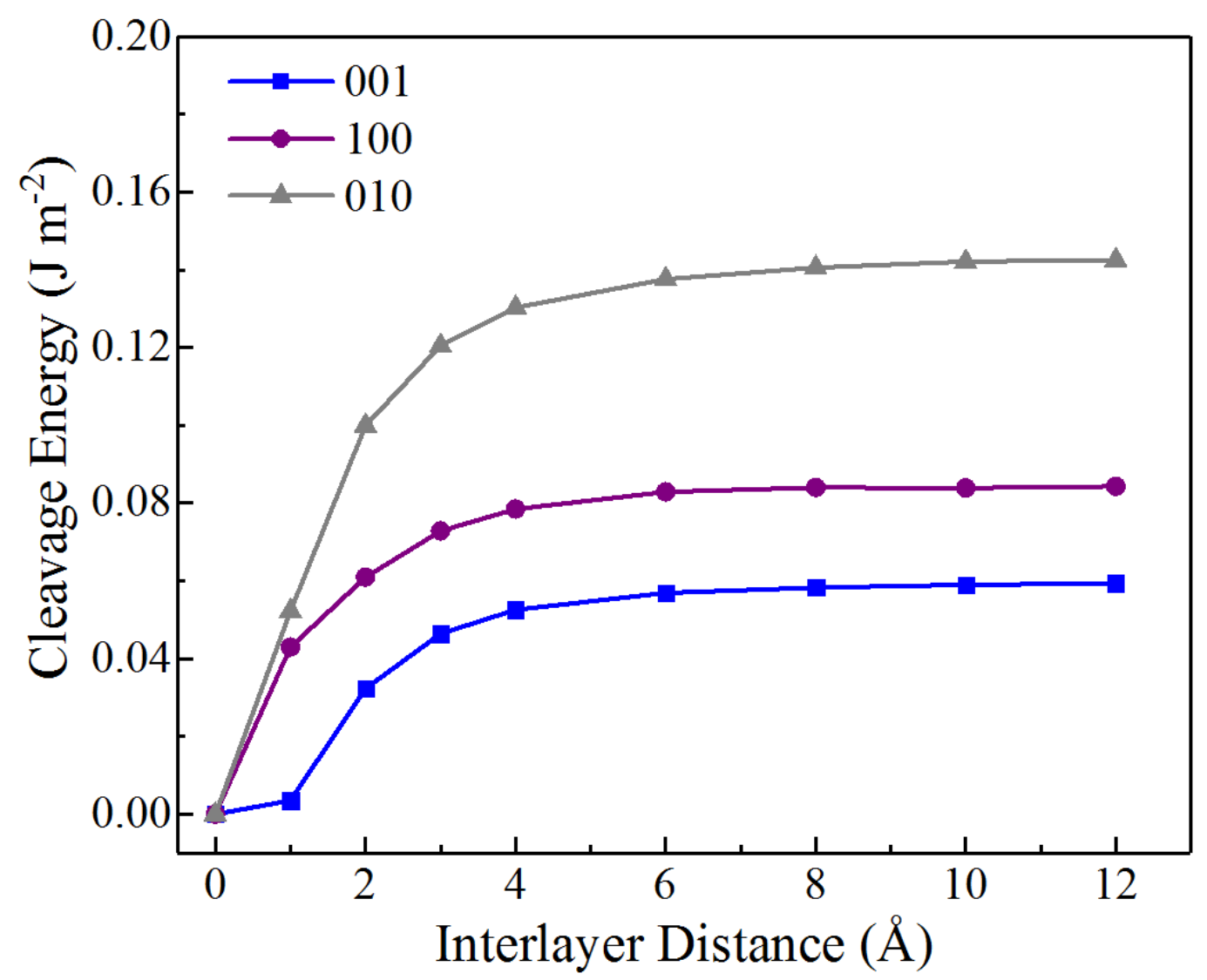

Figure S6. Cleavage energy estimation on the mechanical peeling of DIPAI along the (001), (100) and (010) plane, respectively, by increasing the interlayer distances. The reference starting point (0 A) corresponds to the perfect ferroelectric bulk DIPAI crystal. 
Table S2. Calculated lattice parameters and spontaneous polarization $\left(P_{\mathrm{s}}\right)$ of 2D DIPAC (001) and (100) monolayers, respectively.

\begin{tabular}{llllll}
\hline & monolayer & $a(\AA)$ & $b(\AA)$ & $\gamma\left({ }^{\circ}\right)$ & $P_{\mathrm{s}}\left(10^{-6} \mu \mathrm{C} \mathrm{cm}^{-1}\right)$ \\
\hline \multirow{2}{*}{ DIPAC } & $(001)$ & 7.23 & 7.58 & 90.00 & 1.61 \\
& $(100)$ & 7.24 & 7.33 & 90.00 & 1.54 \\
& $(001)$ & 7.58 & 7.75 & 90.00 & 1.47 \\
DIPAI & $(100)$ & 7.16 & 7.63 & 90.00 & 1.61 \\
\hline
\end{tabular}




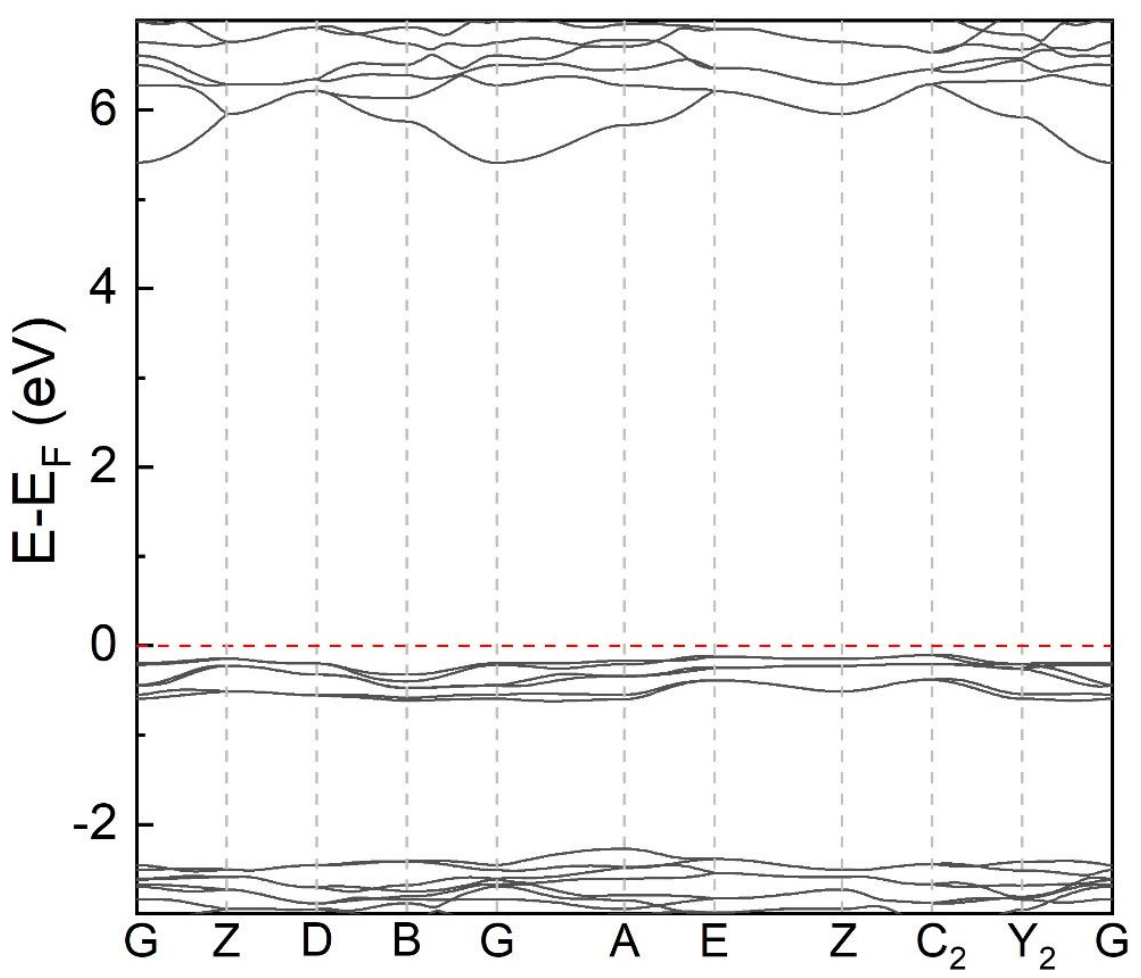

Figure S7. Computed electronic structure of 3D bulk DIPAB molecular crystal. The Fermi level is set to be zero (red dash line). 


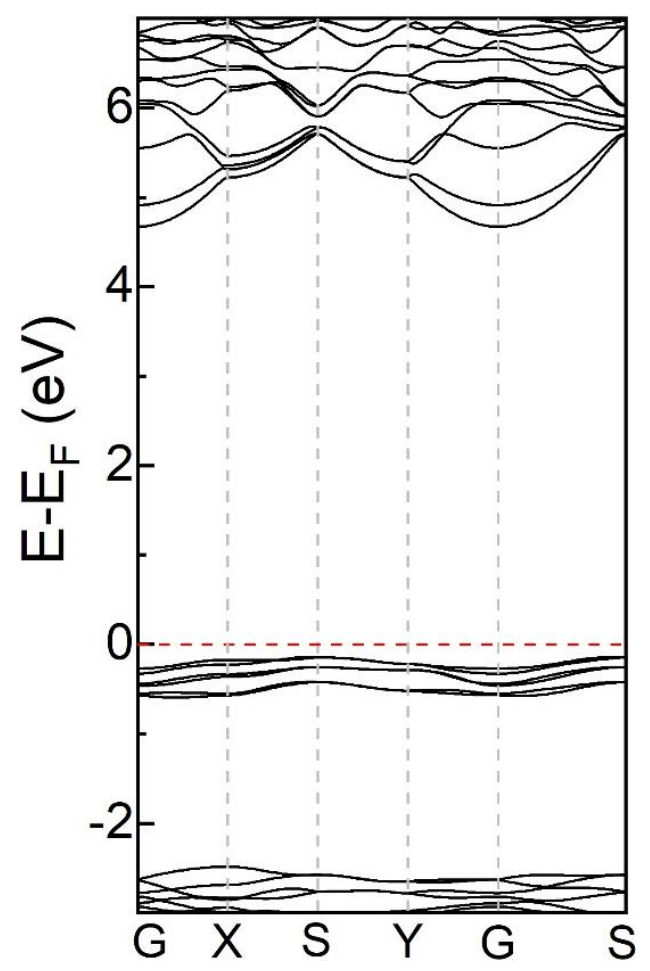

Figure S8. Computed electronic structure of 2D (001) monolayer DIPAB molecular crystal. The Fermi level is set to be zero (red dash line). 


\section{SUPPORTING REFERENCES}

(1) (a) Blöchl, P. E. Projector Augmented-Wave Method. Phys. Rev. B 1994, 50, 17953-17979; (b)

Kresse, G.; Joubert, D. From Ultrasoft Pseudopotentials to the Projector Augmented-Wave Method. Phys. Rev. B 1999, 59, 1758-1775.

(2) Perdew, J. P.; Burke, K.; Ernzerhof, M. Generalized Gradient Approximation Made Simple. Phys. Rev. Lett. 1996, 77, 3865-3868.

(3) (a) Kresse, G.; Furthmüller, J. Efficient Iterative Schemes for Ab Initio Total-Energy Calculations Using a Plane-Wave Basis Set. Phys. Rev. B 1996, 54, 11169-11186; (b) Kresse, G.; Furthmuller, J. Efficiency of Ab-Initio Total Energy Calculations for Metals and Semiconductors Using a Plane-Wave Basis Set. Comput. Mater. Sci. 1996, 6, 15-50.

(4) Monkhorst, H. J.; Pack, J. D. Special Points for Brillouin-Zone Integrations. Phys. Rev. B 1976, 13, $5188-5192$.

(5) (a) Fu, D.-W.; Cai, H.-L.; Liu, Y.; Ye, Q.; Zhang, W.; Zhang, Y.; Chen, X.-Y.; Giovannetti, G.; Capone, M.; Li, J.; Xiong, R.-G. Diisopropylammonium Bromide Is a High-Temperature Molecular Ferroelectric Crystal. Science 2013, 339, 425-428; (b) Fu, D.-W.; Zhang, W.; Cai, H.-L.; Ge, J.-Z.; Zhang, Y.; Xiong, R.-G. Diisopropylammonium Chloride: A Ferroelectric Organic Salt with a High Phase Transition Temperature and Practical Utilization Level of Spontaneous Polarization. Adv. Mater.

2011, 23, 5658-5662.

(6) Grimme, S.; Antony, J.; Ehrlich, S.; Krieg, H. A Consistent and Accurate Ab Initio Parametrization of Density Functional Dispersion Correction (DFT-D) for the 94 Elements H-Pu. J. Chem. Phys. 2010, $132,154104$.

(7) Sun, J.; Ruzsinszky, A.; Perdew, J. P. Strongly Constrained and Appropriately Normed Semilocal Density Functional. Phys. Rev. Lett. 2015, 115, 036402.

(8) Sabatini, R.; Gorni, T.; de Gironcoli, S. Nonlocal Van Der Waals Density Functional Made Simple and Efficient. Phys. Rev. B 2013, 87, 041108.

(9) Peng, H.; Yang, Z.-H.; Perdew, J. P.; Sun, J. Versatile van der Waals Density Functional Based on a Meta-Generalized Gradient Approximation. Phys. Rev. X 2016, 6, 041005.

(10) Allen, M. P.; Tildesley, D. J., Computer Simulation of Liquids. Revised ed.; Oxford University Press: New York, 1991.

(11) (a) Parrinello, M.; Rahman, A. Crystal Structure and Pair Potentials: A Molecular-Dynamics Study. Phys. Rev. Lett. 1980, 45, 1196-1199; (b) Parrinello, M.; Rahman, A. Polymorphic Transitions in Single Crystals: A New Molecular Dynamics Method. J. Appl. Phys. 1981, 52, 7182-7190. 